\title{
Bibliographie der deutsch- und englischsprachigen Wittgenstein-Ausgaben ${ }^{1}$
}

\author{
Alois Pichler, Bergen / Michael A.R. Biggs, Hertfordshire
}

Ab 2002 bearbeitet von Sarah Anna Szeltner, Kassel

\section{Gliederung}

Übersicht zu den Ausgaben mit Quellen im Nachlass . . . . . . . . 250

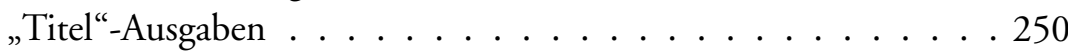

Helsinki-Ausgabe . . . . . . . . . . . . . . . . . . . . . . . . . . . . . . . . . . . . . . .

Teil-Ausgaben . . . . . . . . . . . . . . . . . . . . . . . . . . . . . . . . . . . . .

Wiener Ausgabe . . . . . . . . . . . . . . . . . . . . . . . . . . . . . . . . . . . . . . .

Bergen Electronic Edition (CD-ROM) . . . . . . . . . . . . . . . . . . . . . . .

Bergen Web-Ausgaben . . . . . . . . . . . 253

1 Bibliographie der Sammelausgaben . . . . . . . . . . . . . . . 253

1.1 Nachlass-Faksimile . . . . . . . . . . . . . . . . . . . . . . . . . . . . . . . . .

1.2 Bergen Nachlass-Textausgaben . . . . . . . . . . . . . . . . . . . . . . . . . . . . .

1.3 Suhrkamp-Schriften . . . . . . . . . . . . . . . . . . . . . . . . . . . . . . . . . . .

1.4 Suhrkamp-Werkausgabe . . . . . . . . . . . . . . . . . . . . . . . . . . . . .

1.5 Intelex-Ressource . . . . . . . . . . . . . . . 255

1.6 Wiener Ausgabe . . . . . . . . . . . . . . . . . . . . . . . . . . . . . . . . . . . . . . . . . .

1.7 Helsinki-Ausgabe . . . . . . . . . . . . . . . . . . . . . . . . . . . . . . . . . . . . . . . . .

1.8 Sammelbände . . . . . . . . . . . . . . . . . 257

1.9 Korrespondenz-Sammelausgaben . . . . . . . . . 258

1 Die Bibliographie basiert auf den Bibliographien in: M. Biggs \& A. Pichler: Wittgenstein: Two Source Catalogues and a Bibliography. Catalogues of the $\mathrm{Pu}$ blished Texts and of the Published Diagrams, each Related to its Sources. Working Papers from the Wittgenstein Archives at the University of Bergen 7. Bergen 1993; A. Pichler: Wittgensteins Philosophische Untersuchungen: Vom Buch zum Album. Amsterdam/New York 2004; außerdem den Bibliographien von F.H. Lapointe (1980), S.G. Shanker (1986) und G. Frongia und B. McGuinness (1990). Aktualisierungen bis 2010 von Sarah Anna Szeltner. 
2 Bibliographie der Einzelausgaben . . . . . . . . . . . . 259

2.1 Veröffentlichungen mit Erstveröffentlichung zu Lebzeiten . . 259

2.2 Veröffentlichungen mit Quellen in den Nachlassteilen 101-245 (Manuskripte und Typoskripte) . . . . . . . . . 261

2.3 Veröffentlichungen mit Quellen in den Nachlassteilen 301-311 (Diktate) . . . . . . . . . . . . . . 275

2.4 Veröffentlichungen von Vorlesungs- und Gesprächsaufzeichnungen (Auswahl) . . . . . . . . . . . . . 276

2.5 Veröffentlichungen von Korrespondenz (Auswahl) . . . . . . 281

3 Bibliographie der Veröffentlichungen von Waismanns „Wittgenstein-Arbeiten" (Auswahl) . . . . . . . . . . . . . 286

Übersicht zu den Ausgaben mit Quellen im Nachlass

$$
\text { „Titel“-Ausgaben }{ }^{2}
$$

Deutsch Englisch

AM NM Aufzeichnungen, die G.E. Moore in Norwegen nach Diktat niedergeschrieben hat $(1960,1961,1979)$ [MS 301]

AL NL Aufzeichnungen über Logik $(1957,1960,1979,1984$, 1996) [TS 201a, 201b]

AVES LPE Aufzeichnungen für Vorlesungen über „privates Erlebnis“ und „Sinnesdaten“ $(1968,1989,1993)$ [MSS $148-149,151]$

BBB BBB Das Blaue und das Braune Buch (1958, 1960, 1969, 1970, 1998) [TSS 309-310]

BF RF Bemerkungen über Frazers Golden Bough (1967, 1971, 1979a, 1979b, 1989, 1993) [TS 211, 213, MSS 110, 143]

BGM RFM Bemerkungen über die Grundlagen der Mathematik (1956, 1967, 1974, 1978) [TSS 221-224, MSS 117, $121-122,124-127,164]$

2 In runden Klammern hinter dem Titel sind das Datum der Erstausgabe und evtl. weiterer Ausgaben angegeben. Der Abschnitt in eckigen Klammern enthält die für die Veröffentlichung verwendeten Nachlassquellen; für detaillierte Informationen zu diesen Quellen siehe Pichlers Katalog in Biggs \& Pichler 1993, der auch Seitenund Datierungsangaben enthält. 
BPP RPP Bemerkungen über die Philosophie der Psychologie (Zwei Bände 1980, 1998) [TSS 229, 232, 244-245]

BT BT Big Typescript (2005) [TS 213]

BÜF ROC Bemerkungen über die Farben $(1977,2007)$ [MSS $172-173,176]$

DB MT Denkbewegungen (1997) [MS 183]

EPB - $\quad$ Eine Philosophische Betrachtung (1970) [MS 115]

GB YB Das Gelbe Buch $(1979,1982,1984)$ [TS 311]

GT _ - Geheime Tagebücher (1985a, 1985b, 1991a, 1991b) [MSS 101-103]

LSPP LW Letzte Schriften über die Philosophie der Psychologie (Zwei Bände 1982 und 1992, 1998) [MSS $137-138$, 169-171, 173-174, 176]

PB PR Philosophische Bemerkungen (1964, 1975, 1998)

[TSS 209, 214a, 215a, 215b, MS 109]

PG PG Philosophische Grammatik $(1969,1974,2005)$ [TSS

211, 213, 214a-214c, MSS 112, 114-116, 140]

PH PH Philosophie $(1989,1991,1993)$ [TS 213]

PU PI Philosophische Untersuchungen $(1953,1958,1967$, 1968, 1997, 2001, 2003, 2006, 2007, 2009) [TSS 227, 234. Kritische Ausgabe 2001: TSS 227, 234, MSS 142, 144, TSS 225, 220, 221, 239, 242]

PT PT Prototractatus $(1971,1989,1996)$ [MS 104]

TB NB Tagebücher $(1960,1961,1979,1998)$ [MSS $101-$ 103]

TLP TLP Tractatus Logico-Philosophicus (1921, 1922, 1933, 1955, 1961, 1963, 1972, 1989, 2003, 2004, 2009) [TSS 202-203. Kritische Ausgabe 1989: TSS 202203, MSS 101-104, 301, TSS 201a-b]

ÜG OC Über Gewißheit (1969, 1971, 1974, 2006) [MSS 172, 174-177]

UW CE Ursache und Wirkung: Intuitives Erfassen (1976, 1989, 1993) [MSS 119, 159-160]

VB CV Vermischte Bemerkungen (1977, 1978, 1980, 1984, 1994, 1998, 2006) [MSS 101, 105-113, 116-138, $145-147,153 a-157 b, 162 a-163,165,167-169$, $173-176,179-180 \mathrm{a}]$

VE LE Vortrag über Ethik $(1965,1968,1989,1993,2007)$ [TS 207. Kritische Ausgabe 2007: TS 207, MSS 139a139b] 


\begin{tabular}{|c|c|c|}
\hline WAR & WAR & Wittgenstein an Ramsey $(1967,1980)$ [TS 206] \\
\hline WBG & PDE & $\begin{array}{l}\text { Geleitwort zum Wörterbuch für Volksschulen (1971, } \\
\text { 1977, 1993) [TS 205] }\end{array}$ \\
\hline Z & Z & Zettel $(1967,1981,1984,1998)$ [TS 233] \\
\hline- & NPL & Notes for the „Philosophical Lecture“(1993) [MS 166] \\
\hline - & PIP & Wittgenstein's 1938 Preface (2010) \\
\hline
\end{tabular}

Helsinki-Ausgabe

[Ausgangspunkt für PU 2001]

PUF Philosophische Untersuchungen. Frühversion (1979) [TSS 220, 221, 225, MS 141]

PUZ Philosophische Untersuchungen. Mittelversion (1979) [TSS 227, 242]

239 Philosophische Untersuchungen (TS 239) (1979) [TS 239]

227 Philosophische Untersuchungen I (TS 227) (1981) [TS 227]

\section{Teil-Ausgaben}

1711989 Teile von MS 171 (in Huitfeldt/Rossvær 1989) [MS 171]

1051993 Teile von MS 105 (in Nedo 1993) [MS 105]

1151993 Teile von MS 115 (in Nedo 1993) [MS 115]

1261993 Teile von MS 126 (in Nedo 1993) [MS 126]

1331996 Teile von MS 133 (in Rothhaupt 1996) [MS 133]

1361996 Teile von MS 136 (in Rothhaupt 1996) [MS 136]

1371996 Teile von MS 137 (in Rothhaupt 1996) [MS 137]

1671996 Teile von MS 167 (in Rothhaupt 1996) [MS 167]

1731996 Teile von MS 173 (in Rothhaupt 1996) [MS 173]

1761996 Teile von MS 176 (in Rothhaupt 1996) [MS 176]

2131996 Teile von MS 213 (in Rothhaupt 1996) [MS 213]

\section{Wiener Ausgabe}

Wi1 Wiener Ausgabe Band 1 Philosophische Bemerkungen (1994) [MSS 105-106]

Wi2 Wiener Ausgabe Band 2 Philosophische Betrachtungen,Philosophische Bemerkungen (1994) [MSS 107-108]

Wi3 Wiener Ausgabe Band 3 Bemerkungen, Philosophische Bemerkungen (1995) [MSS 109-110] 
Wi4 Wiener Ausgabe Band 4 Bemerkungen zur Philosophie, Bemerkungen zur philosophischen Grammatik (1995) [MSS 111-112]

Wi5 Wiener Ausgabe Band 5 Philosophische Grammatik (1996) [MSS 113-114]

Wi11 Wiener Ausgabe Band 11 The Big Typescript (2000) [TS 213]

\section{Bergen Electronic Edition (CD-ROM)}

BEE Wittgenstein's Nachlass. The Bergen Electronic Edition (1998-2000) [MSS 101-183, TSS 201-233, 235-245, MS 301, TSS 302-306, 309-310]

\section{Bergen Web-Ausgaben}

115 Machine-readable version text samples: 115 Volume XI. „Philosophische Bemerkungen“ (1996) [MS 115]

201a Machine-readable version text samples: 201a „Notes on Logic“ (1996) [TS 201a]

BTE Wittgenstein Source Bergen Text Edition (2009-, http://www. wittgensteinsource.org/) [MSS 114, 115, 139a, 140(S.40), 141, 148, 149, 150, 152, 153a, 153b, 154, 155, 156a, TSS 201a1, 201a2, 207, $212,213,310]$

BFE Wittgenstein Source Bergen Facsimile Edition (2009-, http://www. wittgensteinsource.org/) [MSS 114, 115, 139a, 140(S.40), 141, 148, 149, 150, 152, 153a, 153b, 154, 155, 156a, TSS 201a1, 201a2, 207, $212,213,310]$

\section{Bibliographie der Sammelausgaben}

\subsection{Nachlass-Faksimile}

- The Wittgenstein Papers. Mikrofilm/Xerokopie. Ithaca, New York: Cornell University Library, Cornell University Microfilms 1968.

- Conservation Microfilm of the Wittgenstein papers. Mikrofilm. Cambridge: Cambridge University Library, Trinity College o. J.

- Mikrofilm der Österreichischen Nationalbibliothek. Cod.Ser.n. 2201822022. Wien: Österreichische Nationalbibliothek, Abt. f. Reprographie 1980.

- Wittgenstein's Nachlass. The Bergen Electronic Edition. Hg. vom Wittgenstein-Archiv an der Universität Bergen unter der Leitung von Claus 
Huitfeldt. Oxford: Oxford University Press 1998-2000. [5 FaksimileCD-ROM; ca. 21.500 Nachlass-Faksimile in jpg-Format; InfoViewProgramm]

- Wittgenstein Source Bergen Faksimile Edition. Hg. von Alois Pichler, unter Mitarbeit von H.W. Krüger, D.C.P. Smith, T.M. Bruvik, A. Lindebjerg, V. Olstad. Bergen: Wittgenstein-Archiv an der Universität Bergen 2009. [http://www.wittgensteinsource.org; Web-Ausgabe von ca. 5000 Nachlass-Faksimile in jpg-Format]

\subsection{Bergen Nachlass-Textausgaben}

- Wittgenstein's Nachlass. The Bergen Electronic Edition. Hg. vom Wittgenstein-Archiv an der Universität Bergen unter der Leitung von Claus Huitfeldt. Oxford: Oxford University Press 1998-2000. [5 FaksimileCD-ROM und 1 Text-CD-ROM. Edition des Nachlasses in zwei Textversionen, „diplomatic“ und „normalized“ (zwei Folio Views Infobases; Folio Views-Programm), und als Faksimile (jpg-Format; InfoView-Programm). Links zwischen den beiden Textversionen und links der beiden Textversionen auf Seite- bzw. Zettel-Ebene zu den Faksimile]

- Wittgenstein Source Bergen Text Edition. Hg. von Alois Pichler, unter Mitarbeit von H.W. Krüger, D.C.P. Smith, T.M. Bruvik, V. Olstad. Bergen: Wittgenstein-Archiv an der Universität Bergen 2009. [http:// www.wittgensteinsource.org; Web-Ausgabe von ca. 5000 Nachlassseiten in in zwei Textversionen, „diplomatisch“ und „normalisiert"]

\subsection{Suhrkamp-Schriften}

- Schriften Band 1. Tractatus logico-philosophicus, Tagebücher 19141916, Philosophische Untersuchungen. Frankfurt am Main: Suhrkamp 1960.

- Schriften Band 2. Philosophische Bemerkungen. Frankfurt am Main: Suhrkamp 1964.

- Schriften Band 3. Wittgenstein und der Wiener Kreis von Friedrich Waismann. Frankfurt am Main: Suhrkamp 1967.

- Schriften Band 4. Philosophische Grammatik. Frankfurt am Main: Suhrkamp 1969.

- Schriften Band 5. Das Blaue Buch, Eine Philosophische Betrachtung, Zettel. Frankfurt am Main: Suhrkamp 1970. 
- Schriften Band 6. Bemerkungen über die Grundlagen der Mathematik. Frankfurt am Main: Suhrkamp 1974.

- Schriften Band 7. Wittgensteins Vorlesungen über die Grundlagen der Mathematik: Cambridge, 1939. Frankfurt am Main: Suhrkamp 1978.

- Schriften Band 8. Bemerkungen über die Philosophie der Psychologie. Frankfurt am Main: Suhrkamp 1982.

\subsection{Suhrkamp-Werkausgabe}

- Werkausgabe Band 1. Tractatus logico-philosophicus, Tagebücher 19141916, Philosophische Untersuchungen. Frankfurt am Main: Suhrkamp 1984. [In der Suhrkamp-Werkausgabe von 1984 ist G.H. von Wright fälschlicherweise als Mitherausgeber der Philosophischen Untersuchungen angegeben. Neuauflage 2009]

- Werkausgabe Band 2. Philosophische Bemerkungen. Frankfurt am Main: Suhrkamp 1984. [Neuauflage 2006]

- Werkausgabe Band 3. Ludwig Wittgenstein und der Wiener Kreis. Frankfurt am Main: Suhrkamp 1984. [Neuauflage 2006]

- Werkausgabe Band 4. Philosophische Grammatik. Frankfurt am Main: Suhrkamp 1984. [Neuauflage 2009]

- Werkausgabe Band 5. Das Blaue Buch, Eine Philosophische Betrachtung (Das Braune Buch). Frankfurt am Main: Suhrkamp 1984. [Neuauflage 2008]

- Werkausgabe Band 6. Bemerkungen über die Grundlagen der Mathematik. Frankfurt am Main: Suhrkamp 1984. [Neuauflage 2009]

- Werkausgabe Band 7. Bemerkungen über die Philosophie der Psychologie, Letzte Schriften über die Philosophie der Psychologie. Frankfurt am Main: Suhrkamp 1984. [Neuauflage 2009]

- Werkausgabe Band 8. Bemerkungen über die Farben, Über Gewißheit, Zettel, Vermischte Bemerkungen. Frankfurt am Main: Suhrkamp 1984. [Neuauflage 2008]

\subsection{Intelex-Ressource}

- Past Masters. The Published Works of Ludwig Wittgenstein. Hg. von InteLex Corporation. Clayton, G.A.: InteLex 1993. [Enthält: Tagebücher, Tractatus Logico-Philosophicus, Philosophische Bemerkungen, Philosophische Grammatik, Bemerkungen über Frazers Golden Bough, Eine 
Philosophische Betrachtung, Ursache und Wirkung: Intuitives Erfassen, Bemerkungen über die Grundlagen der Mathematik, Philosophische Untersuchungen, Zettel, Bemerkungen über die Philosophie der Psychologie (Band 1 und 2), Letzte Schriften über die Philosophie der Psychologie (Band 1), Über Gewißheit, Bemerkungen über die Farben, Vermischte Bemerkungen; Review of P. Coffey's „The Science of Logic“, Notes on Logic, Notes dictated to G.E. Moore in Norway, Some Remarks on Logical Form, A Lecture on Ethics, The Blue and Brown Books, Notes for Lectures on „Private Experience“ and „Sense Data"]

\subsection{Wiener Ausgabe}

- Wiener Ausgabe Band 1. Philosophische Bemerkungen. Hg. von Michael Nedo. Wien, New York: Springer 1994.

- Wiener Ausgabe Band 2. Philosophische Betrachtungen, Philosophische Bemerkungen. Hg. von Michael Nedo. Wien, New York: Springer 1994.

- Wiener Ausgabe Band 3. Bemerkungen, Philosophische Bemerkungen. Hg. von Michael Nedo. Wien, New York: Springer 1995.

- Wiener Ausgabe Band 4. Bemerkungen zur Philosophie, Bemerkungen zur philosophischen Grammatik. Hg. von Michael Nedo. Wien, New York: Springer 1995.

- Wiener Ausgabe Band 5. Philosophische Grammatik. Hg. von Michael Nedo. Wien, New York: Springer 1996.

- Wiener Ausgabe Band 6. Hg. von Michael Nedo. [Erscheinungstermin: März 2015]

- Wiener Ausgabe Band 7. Hg. von Michael Nedo. [Erscheinungstermin: März 2015]

- Wiener Ausgabe Band 10/1. Hg. von Michael Nedo. [Erscheinungstermin: März 2015]

- Wiener Ausgabe Band 10/2. Hg. von Michael Nedo. [Erscheinungstermin: März 2015]

- Wiener Ausgabe Band 11. The Big Typescript / Typoskript 213. Hg. von Michael Nedo. Wien, New York: Springer 2000.

- Wiener Ausgabe Vorträge, Diktate, Tagebücher. Hg. von Michael Nedo. [Erscheinungstermin: März 2015]

- Wiener Ausgabe Apparatus. Konkordanz zu den Bänden 1-5. Hg. von Michael Nedo. Wien, New York: Springer 1997.

- Wiener Ausgabe Apparatus. Konkordanz zu Band 6. Hg. von Michael Nedo. [Erscheinungstermin: März 2015] 
- Wiener Ausgabe Apparatus Umarbeitung der Synopsen zu den Manuskriptbänden I-IV (TS 209). Hg. von Michael Nedo. [Erscheinungstermin: März 2015]

- Wiener Ausgabe Apparats Synopse der Bände V-X. Hg. von Michael Nedo. [Erscheinungstermin: März 2015]

- Wiener Ausgabe Apparats Synopse der Manuskriptbände V-X. Hg. von Michael Nedo. Wien, New York: Springer 2000.

- Wiener Ausgabe Apparatus. Register zu den Bänden 1-5. Hg. von Michael Nedo. Wien, New York: Springer 1998.

\subsection{Helsinki-Ausgabe}

[Ausgangspunkt für PU 2001]

- Philosophische Untersuchungen. Frühversion 1937-1938. TS225, TS220, TS 221 mit MS 141 als Anhang. Mit Kommentaren. Hg. von G.H. von Wright und Heikki Nyman. Helsinki 1979.

- Philosophische Untersuchungen. ,Mittelversion'(1945). Mit Kommentaren. Hg. von G.H. von Wright und Heikki Nyman. Helsinki 1979.

- Philosophische Untersuchungen. TS 239. Edierter Text mit Kommentaren. Hg. von G.H. von Wright und Heikki Nyman. Helsinki 1979.

- Ludwig Wittgenstein. Philosophische Untersuchungen I (TS 227). Der edierte Text mit Kommentaren und Anhängen. Hg. von G.H. von Wright und Heikki Nyman. Helsinki 1981.

\subsection{Sammelbände}

- Vortrag über Ethik und andere kleine Schriften. Hg. und übersetzt von Joachim Schulte. Frankfurt am Main: Suhrkamp 1989. [Enthält: Vortrag über Ethik, Bemerkungen über logische Form, Bemerkungen über Frazers Golden Bough, Aufzeichnungen für Vorlesungen über „privates Erlebnis“ und „Sinnesdaten“, Ursache und Wirkung. Intuitives Erfassen. Neuauflage 2007]

- Philosophical Occasions 1912-1951. Hg. und eingeleitet von James C. Klagge und Alfred Nordmann. Indianapolis and Cambridge, USA: Hackett 1993. [Enthält: Book Review of P. Coffey's The Science of Logic, Some Letters of Wittgenstein. Ludwig Wittgenstein and William Eccles, Geleitwort zum Wörterbuch für Volksschulen, Some Remarks on Logical 
Form, A Lecture on Ethics, Wittgenstein's Lectures in 1930-33, Bemerkungen über Frazers Golden Bough, Letters to the Editor of Mind, Philosophie, Notes for Lectures on "Private Experience“ and „Sense Data", The Language of Sense Data and Private Experience, Ursache und Wirkung: Intuitives Erfassen, Lectures on Freedom of the Will, Notes for the „Philosophical Lecture“, Letters from Ludwig Wittgenstein to Georg Henrik von Wright]

- Vier Originalmanuskripte, darunter die Hauptwerke. Wien: Inlibris 2003. [Aus dem Nachlass von Rudolf Koder. Enthält die Logisch-philosophische Abhandlung, die Philosophischen Untersuchungen, das Redemanuskript der Lectures on Ethics und Wittgensteins Tagebücher aus den Jahren 1930-32 und 1936-37]

- Major Works: Selected Philosophical Writings. New York: Harper Perennial 2009. [Featuring the complete texts of Tractatus Logico-Philosophicus, The Blue and Brown Books: Studies for Philosophical Investigations, and On Certainty. Copyright für The Blue and the Brown Books: 1958 by Basil Blackwell; für On Certainty: 1969 by Basil Blackwell]

\subsection{Korrespondenz-Sammelausgaben}

- Briefe. Hg. von B.F. McGuinness und G.H. von Wright, übersetzt von Joachim Schulte. Frankfurt am Main: Suhrkamp 1980.

- Public and Private Occasions. Hg. von James C. Klagge und Alfred Nordmann. Lanham, Boulder, New York, Oxford: Rowman and Littlefield 2003.

- Briefwechsel. Innsbrucker elektronische Ausgabe. Hg. von Monika Seekircher, Anton Unterkircher und Brian McGuinness. Charlottesville, Virginia: Intelex (Web-Ausgabe) 2004.

- Wittgenstein in Cambridge. Letters and Documents, 1911-1951. Hg. von Brian McGuinness. Malden (Mass.): Blackwell 2008. 
Bibliographie der deutsch- und englischsprachigen Wittgenstein-Ausgaben

\section{Bibliographie der Einzelausgaben ${ }^{3}$}

\subsection{Veröffentlichungen mit Erstveröffentlichung zu Lebzeiten}

RC 1913 „Review of P. Coffey's, The Science of Logic“". In: The Cambridge Review 34 p.351 (Cambridge 1913). [Englisch. Erste Veröffentlichung auf Deutsch RC 1988]

TLP 1921 „Logisch-philosophische Abhandlung“. In: Annalen der Natur-und Kulturphilosophie 14S. 184-262 (Leipzig 1921). Hg. von W. Ostwald. [Deutsch und deutsche Übersetzung von Bertrand Russells Einleitung. Erste Veröffentlichung auf Englisch TLP 1922]

TLP 1922 Tractatus Logico-Philosophicus Hg. von C.K. Ogden, übersetzt von C.K. Ogden und F.P. Ramsey. International Library of Psychology, Philosophy and Scientific Method. London: Kegan Paul, Trench, Trubner. [Mit einer Einleitung auf Englisch von Bertrand Russell. Deutsch mit englischer Übersetzung]

WB 1926 Wörterbuch für Volksschulen. Wien: Hölder-Pichler-Tempsky. [Deutsch]

RLF 1929 "Some Remarks on Logical Form“. In: Proceedings of the Aristotelian Society Supplementary Volume 9, S. 162-171 (London 1929). [Englisch. Erste Veröffentlichung auf Deutsch RLF 1989]

TLP 1933 Tractatus Logico-Philosophicus. Hg. von C.K. Ogden, übersetzt von C.K. Ogden und F.P. Ramsey. Reprinted with a few corrections. International Library of Psychology, Philosophy and Scientific Method. London: Routledge and Kegan Paul. [Einleitung auf Englisch von Bertrand Russell. Deutsch mit englischer Übersetzung]

CEM 1933 „Letter to the Editor“. In: Mind 42 S. 415-416 (Oxford 1933). [Englisch]

TLP 1955 Tractatus Logico-Philosophicus. Hg. von C.K. Ogden, übersetzt von C.K. Ogden und F.P. Ramsey. International Library of Psychology, Philosophy and Scientific Method. London:

3 Die Bibliographie enthält die jeweilige Erstveröffentlichung, die jeweiligen Erstausgaben in Deutsch und Englisch und die Neuausgaben in Deutsch und Englisch. Die Veröffentlichungen in der Suhrkamp-Werkausgabe von 1984 sind insofern angeführt, als sie Erstausgaben oder Neuausgaben darstellen. 
Routledge and Kegan Paul. [Mit einem Register von Max Black. Einleitung auf Englisch von Bertrand Russell. Deutsch mit englischer Übersetzung]

TLP 1961 Tractatus Logico-Philosophicus. Übersetzt von D.F. Pears und B.F. McGuinness. International Library of Philosophy and Scientific Method. London: Routledge and Kegan Paul. [Mit einem Register von den Übersetzern. Einleitung auf Englisch von Bertrand Russell und Deutsch mit englischer Übersetzung]

TLP 1963 Tractatus Logico-Philosophicus. Übersetzt von D.F. Pears und B.F. McGuinness. International Library of Philosophy and Scientific Method. Second impression, with a few corrections. London: Routledge and Kegan Paul. [Mit einem Register von den Übersetzern. Einleitung auf Englisch von Bertrand Russell und Deutsch mit englischer Übersetzung]

TLP 1972 Tractatus Logico-Philosophicus. Übersetzt von D.F. Pears und B.F. McGuinness. Reprinted with corrections in the light of the publication of Wittgenstein's correspondence with C.K. Ogden about the first translation. London: Routledge and Kegan Paul. [Mit einem Register von den Übersetzern. Einleitung auf Englisch von Bertrand Russell und Deutsch mit englischer Übersetzung]

RC 1988 Deutsche Übersetzung von „Review of P. Coffey’s, The Science of Logic“". In: McGuinness, B. Wittgensteins frühe Jahre S. 272-274. Übersetzt von J. Schulte. Frankfurt am Main: Suhrkamp. [Deutsche Übersetzung]

RLF 1989 „Bemerkungen über logische Form“. In: Vortrag über Ethik S. 20-28. Hg. und übersetzt von Joachim Schulte. Frankfurt am Main: Suhrkamp. [Deutsche Übersetzung]

TLP 1989 Logisch-philosophische Abhandlung. Tractatus logico-philosophicus $\mathrm{Hg}$. von Brian McGuinness und Joachim Schulte. Kritische Edition. Frankfurt am Main: Suhrkamp. [Mit einem Register und einer Tabelle von Entsprechungen zwischen AM 1979, NL 1979 und AM und NL in Band 1 der SuhrkampWerkausgabe von 1984. Deutsch und Englisch, Bertrand Russells Einleitung auf Englisch mit deutscher Übersetzung] 
CEM 1993 „Letters to the Editor of Mind“. In: Ludwig Wittgenstein. Philosophical Occasions 1912-1951 S. 156-157. Hg. und eingeleitet von James C. Klagge und Alfred Nordmann. Indianapolis and Cambridge, USA: Hackett. [Englisch]

RC 1993 „Book Review of P. Coffey's, The Science of Logic“". In: Ludwig Wittgenstein. Philosophical Occasions 1912-1951 S. 1-3. Hg. und eingeleitet von James C. Klagge und Alfred Nordmann. Indianapolis and Cambridge, USA: Hackett. [Englisch]

RLF 1993 „Some Remarks on Logical Form“. In: Ludwig Wittgenstein. Philosophical Occasions 1912-1951 S. 28-35. Hg. und eingeleitet von James C. Klagge und Alfred Nordmann. Indianapolis and Cambridge, USA: Hackett. [Englisch]

2.2 Veröffentlichungen mit Quellen in den Nachlassteilen 101-245 (Manuskripte und Typoskripte)

TLP 1921 „Logisch-philosophische Abhandlung“. In: Annalen der Natur-und Kulturphilosophie 14S. 184-262 (Leipzig 1921). Hg. von W. Ostwald. [Deutsch und deutsche Übersetzung von Bertrand Russells Einleitung. Erste Veröffentlichung auf Englisch TLP 1922]

TLP 1922 Tractatus Logico-Philosophicus. Hg. von C.K. Ogden, übersetzt von C.K. Ogden und F.P. Ramsey. International Library of Psychology, Philosophy and Scientific Method. London: Kegan Paul, Trench, Trubner. [Mit einer Einleitung auf Englisch von Bertrand Russell. Deutsch mit englischer Übersetzung]

TLP 1933 Tractatus Logico-Philosophicus. Hg. von C.K. Ogden, übersetzt von C.K. Ogden und F.P. Ramsey. Reprinted with a few corrections. International Library of Psychology, Philosophy and Scientific Method. London: Routledge and Kegan Paul. [Einleitung auf Englisch von Bertrand Russell. Deutsch mit englischer Übersetzung]

PI 1953 Philosophical Investigations / Philosophische Untersuchungen. Hg. von G.E.M. Anscombe und R. Rhees, übersetzt von G.E.M. Anscombe. Oxford: Basil Blackwell. [Deutsch mit englischer Übersetzung] 
TLP 1955 TractatusLogico-Philosophicus. Hg. von C.K. Ogden, übersetzt von C.K. Ogden und F.P. Ramsey. International Library of Psychology, Philosophy and Scientific Method. London: Routledge and Kegan Paul. [Mit einem Register von Max Black. Einleitung auf Englisch von Bertrand Russell. Deutsch mit englischer Übersetzung]

RFM 1956 Remarks on the Foundations of Mathematics/Bemerkungen über die Grundlagen der Mathematik. Hg. von G.H. von Wright, R. Rhees und G.E.M. Anscombe, übersetzt von G.E.M. Anscombe. Oxford: Basil Blackwell. [Mit einem ausführlichen Inhaltsverzeichnis und einem Register. Deutsch mit englischer Übersetzung]

NL 1957 „Notes on Logic“. In: The Journal of Philosophy 54 S. 230245 (New York, USA, 1957). Hg. mit einer Einleitung von H.T. Costello. [Costello-Version. Englisch. Erste Veröffentlichung auf Deutsch NL 1960]

PI 1958 Philosophical Investigations / Philosophische Untersuchungen. Hg. von G.E.M. Anscombe und R. Rhees, übersetzt von G.E.M. Anscombe. Second edition. Oxford: Basil Blackwell. [Deutsch mit englischer Übersetzung]

NL 1960 „Aufzeichnungen über Logik“. Hg. von G.E.M. Anscombe und G.H. von Wright, übersetzt von Günther Patzig und Eberhard Bubser. In: Schriften Bd. 1 S. 186-225. Frankfurt am Main: Suhrkamp. [Costello-Version. Englisch mit deutscher Übersetzung]

TB 1960 „Tagebücher 1914-1916“. Hg. von G.E.M. Anscombe und G.H. von Wright. In: Schriften Bd. 1 S. 85-185. Frankfurt am Main: Suhrkamp. [Deutsch. Erste Veröffentlichung auf Englisch TB 1961]

TB 1961 „Notebooks 1914-1916“. In: Notebooks 1914-1916 S. 2-91. Hg. von G.H. von Wright und G.E.M. Anscombe, übersetzt von G.E.M. Anscombe. Oxford: Basil Blackwell. [Deutsch mit englischer Übersetzung]

PB 1964 Philosophische Bemerkungen. Hg. von Rush Rhees. Oxford: Basil Blackwell. [Mit einem ausführlichen Inhaltsverzeichnis. Deutsch. Veröffentlicht als Schriften Bd. 2 im selben Jahr. Erste Veröffentlichung auf Englisch PB 1975]

LE 1965 „A Lecture on Ethics“. In: „Wittgenstein's Lecture on Ethics“. In: The Philosophical Review 74 S. 3-12 (Ithaca, USA, 1965). [Englisch. Erste Veröffentlichung auf Deutsch LE 1968] 
WAR 1967 „Wittgenstein an Ramsey“. In: Ludwig Wittgenstein und der Wiener Kreis S. 189-191. Hg. von Brian McGuinness. Oxford: Basil Blackwell. [Englisch. Veröffentlicht in Schriften Bd. 3 S. 189-191 im selben Jahr. Erste Veröffentlichung auf Deutsch WAR 1980]

GB 1967 „Bemerkungen über Frazers The Golden Bough“. In: Synthese 17 S. 233-253 (Dordrecht, Holland, 1967). Hg. von R. Rhees. [Deutsch. Erste Veröffentlichung auf Englisch GB 1971]

PI 1967 Philosophical Investigations / Philosophische Untersuchungen. Hg. von G.E.M. Anscombe und R. Rhees, übersetzt von G.E.M. Anscombe. Reprint of the second edition with an index. Oxford: Basil Blackwell. [Mit einem Register zum deutschen Text von C. Schwarck, fußend auf dem Register von Garth Hallett zur englischen Übersetzung. Deutsch mit englischer Übersetzung]

RFM 1967 Remarks on the Foundations of Mathematics/Bemerkungen über die Grundlagen der Mathematik. Hg. von G.H. von Wright, R. Rhees und G.E.M. Anscombe, übersetzt von G.E.M. Anscombe. Second edition. Oxford: Basil Blackwell. [Mit einem ausführlichen Inhaltsverzeichnis und einem Register. Deutsch mit englischer Übersetzung]

Z 1967 Zettel / Zettel. Hg. von G.E.M. Anscombe und G.H. von Wright, übersetzt von G.E.M. Anscombe. Oxford: Basil Blackwell. [Deutsch mit englischer Übersetzung]

LE 1968 „Ethik. Ein Vortrag von Ludwig Wittgenstein“. In: Neue Zürcher Zeitung 2714 January 1968, S. 49-50 (Zürich, Schweitz, 1968). Übersetzt von Franz Wurm. [Deutsche Übersetzung. Eine deutsche Übersetzung der „Lecture on Ethics"von Franz Wurm und Auszüge von Gespächen Wittgensteins mit Friedrich Waismann, Moritz Schlick und Rush Rhees wurden am 7. April 1967 von Radio DRS, Zürich, gesendet. Ein Dank an Dr. Richard Weihe vom English Seminar, Universität Zürich, für diese Information]

LPE 1968 „Wittgenstein's Notes for Lectures on ,Private Experience' and ,Sense Data““. In: The Philosophical Review 77 S. 271-320 (Ithaca, USA, 1968). Hg. von Rush Rhees. [Englisch und Deutsch mit englischer Übersetzung. Erste Veröffentlichung auf Deutsch LPE 1989] 
PI 1968 Philosophical Investigations. Hg. von G.E.M. Anscombe und R. Rhees, übersetzt von G.E.M. Anscombe. Third edition with index. Oxford: Basil Blackwell. [Mit einem Register von Garth Hallett. Englische Übersetzung]

OC 1969 On Certainty / Über Gewißheit. Hg. von G.E.M. Anscombe und G.H. von Wright, übersetzt von Denis Paul und G.E.M. Anscombe. Oxford: Basil Blackwell. [Deutsch mit englischer Übersetzung]

PG 1969 Philosophische Grammatik. Hg. von Rush Rhees. Oxford: Basil Blackwell. [Mit einem ausführlichen Inhaltsverzeichnis. Deutsch. Veröffentlicht als Schriften Bd. 4 im selben Jahr. Erste Veröffentlichung auf Englisch PG 1974]

EPB 1970 „Eine Philosophische Betrachtung“. In: Schriften Bd. 5 S. 117-282. Hg. von R. Rhees, übersetzt von Petra von Morstein. Frankfurt am Main: Suhrkamp. [Deutsch und deutsche Übersetzung]

OC 1971 Über Gewißheit. Hg. von G.E.M. Anscombe und G.H. von Wright. Frankfurt am Main: Suhrkamp. [Mit einem Register. Deutsch]

GB 1971 „Remarks on Frazer's Golden Bough“. Hg. von R. Rhees, übersetzt von A.C. Miles und R. Rhees. The Human World 3 S. 18-41 (Swansea, 1971). [Englische Übersetzung. S. $18-$ 28 Einleitung auf Englisch von Rush Rhees]

PT 1971 Prototractatus. An early version of Tractatus Logico-Philosophicus. Edited by B.F. McGuinness, T. Nyberg and G.H. von Wright, with a translation by D.F. Pears and B.F. McGuinness, an historical introduction by G.H. von Wright and a facsimile of the author's manuscript. London: Routledge and Kegan Paul. [Mit Tabellen zu Entsprechungen zwischen „Prototractatus" und „Tractatus" und „Note on variations between the different editions of the Tractatus". Englisch und Deutsch mit englischer Übersetzung]

TLP 1972 Tractatus Logico-Philosophicus. Übersetzt von D.F. Pears und B.F. McGuinness. Reprinted with corrections in the light of the publication of Wittgenstein's correspondence with C.K. Ogden about the first translation. London: Routledge and Kegan Paul. [Mit einem Register von den Übersetzern. Einleitung auf Englisch von Bertrand Russell und Deutsch mit englischer Übersetzung] 
OC 1974 On Certainty / Über Gewißheit. Hg. von G.E.M. Anscombe und G.H. von Wright, übersetzt von Denis Paul und G.E.M. Anscombe. Reprinted with corrections and indices. Oxford: Basil Blackwell. [Mit einem Register zum deutschen Text von Rush Rhees und einem Register zur englischen Übersetzung von Garth Hallett. Deutsch mit englischer Übersetzung]

PG 1974 Philosophical Grammar. Hg. von Rush Rhees, übersetzt von Anthony Kenny. Oxford: Basil Blackwell. [Mit einem ausführlichen Inhaltsverzeichnis und Corrigenda zu PG 1969. Englische Übersetzung]

RFM 1974 „Bemerkungen über die Grundlagen der Mathematik“. In: Schriften Bd. 6. Hg. von G.H. von Wright, Rush Rhees und G.E.M. Anscombe. Erweiterte und revidierte Neuausgabe. Frankfurt am Main: Suhrkamp. [Mit einem ausführlichen Inhaltsverzeichnis und einem Register. Deutsch. Erste Veröffentlichung auf Englisch RFM 1978]

PB 1975 Philosophical Remarks. Hg. von Rush Rhees, übersetzt von Raymond Hargreaves und Roger White. Oxford: Basil Blackwell. [Mit einem ausführlichen Inhaltsverzeichnis und Corrigenda zu PB 1964. Englische Übersetzung. Lapointe 1980 und Shanker 1986 schreiben die erste englische Übersetzung fälschlicherweise G.E.M. Anscombe, 1968, zu]

UW 1976 „Ursache und Wirkung: Intuitives Erfassen/Cause and Effect: Intuitive Awareness". Hg. von Rush Rhees, übersetzt von P. Winch. Philosophia 6 S. 392-445 (Ramat-Gan, Israel 1976). [Englisch und Deutsch mit englischer Übersetzung. Erste Veröffentlichung auf Deutsch UW 1989]

ROC 1977 Remarks on Colour / Bemerkungen über die Farben. Hg. von G.E.M. Anscombe, übersetzt von Linda L. McAlister und Margarete Schättle. Oxford: Basil Blackwell. [Deutsch mit englischer Übersetzung]

VB 1977 Vermischte Bemerkungen. Hg. von Georg Henrik von Wright unter Mitarbeit von Heikki Nyman. Frankfurt am Main: Suhrkamp. [Mit Registern. Deutsch, im selben Jahr veröffentlicht bei Basil Blackwell]

WBG 1977 „Geleitwort zum Wörterbuch für Volksschulen / [Author's] Preface to the Wörterbuch für Volksschulen“. In: Wörterbuch für Volksschulen S.xxv-xxxv. Hg. von A. Hübner, E. Leinfellner und W. Leinfellner, übersetzt von E. Leinfellner. Mit einer Einleitung von A. Hübner. Schriften der Österreichischen 
Wittgensteingesellschaft. Wien: Hölder-Pichler-Tempsky. [Deutsch mit englischer Übersetzung]

RFM 1978 Remarks on the Foundations of Mathematics. Hg. von G.H. von Wright, R. Rhees und G.E.M. Anscombe, übersetzt von G.E.M. Anscombe. Third edition, revised and reset. Oxford: Basil Blackwell. [Mit einem ausführlichen Inhaltsverzeichnis und einem Register. Englische Übersetzung]

VB 1978 Vermischte Bemerkungen. Hg. von G.H. von Wright unter Mitarbeit von Heikki Nyman. Zweite Auflage. Frankfurt am Main: Suhrkamp. [Mit einem Register. Deutsch, im selben Jahr veröffentlicht bei Basil Blackwell. Erste Veröffentlichung auf Englisch in VB 1980]

GB 1979a Remarks on Frazer's Golden Bough / Bemerkungen über Frazers Golden Bough. Hg. von Rush Rhees, übersetzt von A.C. Miles und Rush Rhees. Doncaster: The Brynmill Press. [Deutsch mit englischer Übersetzung]

GB 1979b „Remarks on Frazer’s Golden Bough“. In: Wittgenstein. Sources and Perspectives S. 61-81. Hg. von C.G. Luckhardt, übersetzt von John Beversluis. Hassocks, Sussex: The Harvester Press. [Englische Übersetzung]

NL 1979 „Notes on Logic“. In: Notebooks 1914-1916S. 93-107. Hg. von G.H. von Wright und G.E.M. Anscombe, übersetzt von G.E.M. Anscombe. Second edition. Oxford: Basil Blackwell. [Mit einem Register von E.D. Klemke und Ali Enayat. RussellVersion. Englisch. Erste Veröffentlichung auf Deutsch NL 1984]

TB 1979 „Notebooks 1914-1916“. In: Notebooks 1914-1916 S. 2-91. Hg. von G.H. von Wright und G.E.M. Anscombe, übersetzt von G.E.M. Anscombe. Second edition. Oxford: Basil Blackwell. [Mit einem Register von E.D. Klemke und Ali Enayat. Deutsch mit englischer Übersetzung]

WAR 1980 „Wittgenstein an Ramsey“. In: Briefe S. 160-161. Hg. von B.F. McGuinness und G.H. von Wright, übersetzt von J. Schulte. Frankfurt am Main: Suhrkamp. [Englisch mit deutscher Übersetzung]

RPP 1980a Remarks on the Philosophy of Psychology/Bemerkungen über die Philosophie der Psychologie. Bd. 1. Hg. von G.E.M. Anscombe und G.H. von Wright, übersetzt von G.E.M. Anscombe. Oxford: Basil Blackwell. [Mit einem Register von Heikki Nyman. Deutsch mit englischer Übersetzung] 
RPP 1980b Remarks on the Philosophy of Psychology/Bemerkungen über die Philosophie der Psychologie. Bd. 2. Hg. von G.H. von Wright und Heikki Nyman, übersetzt von C.G. Luckhardt und Maximilian A.E. Aue. Oxford: Basil Blackwell. [Mit einem Register und Tabellen von Entsprechungen zwischen TS 232 und Z, TS 232 und PI, TS 232 und VB. Deutsch mit englischer Übersetzung]

VB 1980 Cultureand Value/Vermischte Bemerkungen. Hg.von G.H.von Wright unter Mitarbeit von Heikki Nyman, übersetzt von Peter Winch. Amended second edition. Oxford: Basil Blackwell. [Mit Registern. Deutsch mit englischer Übersetzung]

Z 1981 Zettel. Hg. von G.E.M. Anscombe und G.H. von Wright, übersetzt von G.E.M. Anscombe. Second edition. Oxford: Basil Blackwell. [Mit einem Register von Stephen Amdur. Englische Übersetzung. Erste Veröffentlichung auf Deutsch Z 1984]

LW 1982 Last Writings on the Philosophy of Psychology / Letzte Schriften über die Philosophie der Psychologie. Bd. 1. Hg. von G.H. von Wright und Heikki Nyman, übersetzt von C.G. Luckhardt und Maximilian A.E. Aue. Oxford: Basil Blackwell. [Mit einem Register. Deutsch mit englischer Übersetzung]

NL 1984 „Aufzeichnungen über Logik“. In: Werkausgabe Bd. 1 S. 188208. Hg. von G.H. von Wright und G.E.M. Anscombe, übersetzt von J. Schulte. Frankfurt am Main: Suhrkamp. [Russell-Version. Deutsche Übersetzung.]

VB 1984 „Vermischte Bemerkungen“. In: Werkausgabe Bd. 8 S. 445575. Hg. von G.H. von Wright. Unter Mitarbeit von Heikki Nyman. Frankfurt am Main: Suhrkamp. [Mit einem Register. Deutsch]

Z 1984 „Zettel“. In: Werkausgabe Bd. 8 S. 259-443.Hg. von G.E.M. Anscombe und G.H. von Wright. Zweite Auflage. Frankfurt am Main: Suhrkamp. [Deutsch]

GT 1985a „Diaris Secrets / Geheime Tagebücher“. In: Saber 5 (September) S. 24-49 (Barcelona, Spanien, 1985). Hg. von W. Baum, übersetzt von A. Sanchez Pascual. [Deutsch mit katalanischer Übersetzung]

GT 1985b „Diarios Secretos (y II) / Geheime Tagebücher“. In: Saber 6 (November) S. 30-59 (Barcelona, Spanien, 1985). Hg. von 
GB 1989 „Bemerkungen über Frazers Golden Bough“. In: Vortrag über Ethik S. 29-46. Hg. und übersetzt von Joachim Schulte. Frankfurt am Main: Suhrkamp. [Deutsch]

LE 1989 „Vortrag über Ethik“. In: Vortrag über Ethik S. 9-19. Hg. und übersetzt von Joachim Schulte. Frankfurt am Main: Suhrkamp. [Deutsche Übersetzung]

LPE 1989 „Aufzeichnungen für Vorlesungen über,privates Erlebnis‘ und ,Sinnesdaten“". In: Vortrag über Ethik S. 47-100. Hg. und übersetzt von Joachim Schulte. Frankfurt am Main: Suhrkamp. [Deutsch und deutsche Übersetzung]

UW 1989 „Ursache und Wirkung. Intuitives Erfassen“. In: Vortrag über Ethik S. 101-139. Hg. und übersetzt von Joachim Schulte. Frankfurt am Main: Suhrkamp. [Deutsch und deutsche Übersetzung]

1711989 „Sample printouts of text, indexes and word-lists from transcription of catalogue no 171“. Transkription von Øyvind Brotnov, et al. In: The Norwegian Wittgenstein Project Report 1988 S. $107-170$. Bergen, Norway: The Norwegian Computing Centre for the Humanities. [Mit einem Facsimile von MS 171 aus der Cornell-Kopie. Mit Registern und Wortlisten. Deutsch. Erste Veröffentlichung auf Englisch 171 1992]

PH 1989 „Philosophie“. In: Revue Internationale de Philosophie 43 S. 172-203 (Brussels, Belgium, 1989). Hg. von Heikki Nyman. [\$\$86-93 von TS 213. Erste Veröffentlichung auf Englisch PH 1991]

PT 1989 In: Logisch-philosophische Abhandlung. Tractatus logico-philosophicus S. 180-255. Hg. von Brian McGuinness und Joachim Schulte. Kritische Edition. Frankfurt am Main: Suhrkamp. [„Synoptische“Ausgabe. Mit einem Register und einer Tabelle von Entsprechungen zwischen AM 1979, NL 1979 und AM und NL in Band 1 der Suhrkamp-Werkausgabe von 1984. Deutsch und Englisch, Bertrand Russells Einleitung auf Englisch mit deutscher Übersetzung]

TLP 1989 In: Logisch-philosophische Abhandlung. Tractatus logico-philosophicus S. 1-179. Hg. von Brian McGuinness und Joachim Schulte. Kritische Edition. Frankfurt am Main: Suhrkamp. [„Synoptische“Ausgabe. Mit einem Register und einer Tabelle von Entsprechungen zwischen AM 1979, NL 1979 und AM 
und NL in Band 1 der Suhrkamp-Werkausgabe von 1984. Deutsch und Englisch, Bertrand Russells Einleitung auf Englisch mit deutscher Übersetzung]

GT 1991a Geheime Tagebücher. Hg. von Wilhelm Baum. Wien: Turia und Kant. [Deutsch]

GT 1991b Geheime Tagebücher. Hg. von Wilhelm Baum. Zweite Auflage. Wien: Turia und Kant. [Deutsch]

PH 1991 „Philosophy“. In: Synthese 87 S. 3-22 (Dordrecht, Holland, 1991). Hg. von Heikki Nyman, übersetzt von C.G. Luckhardt und M.A.E. Aue. [Englische Übersetzung von TS 213:\$\$8693]

LW 1992 Last Writings on the Philosophy of Psychology / Letzte Schriften über die Philosophie der Psychologie. Bd. 2. Hg. von G.H. von Wright und Heikki Nyman, übersetzt von C.G. Luckhardt und Maximilian A.E. Aue. Oxford: Basil Blackwell. [Mit einem Register von den Übersetzern. Deutsch mit englischer Übersetzung]

GB 1993 „Remarks on Frazer’s Golden Bough / Bemerkungen über Frazers Golden Bough". In: Ludwig Wittgenstein. Philosophical Occasions 1912-1951 S. 115-155. Hg. und eingeleitet von James C. Klagge und Alfred Nordmann. Indianapolis and Cambridge, USA: Hackett. [Deutsch mit englischer Übersetzung]

LE 1993 „A Lecture on Ethics“. In: Ludwig Wittgenstein. Philosophical Occasions 1912-1951 S. 36-44. Hg. und eingeleitet von James C. Klagge und Alfred Nordmann. Indianapolis and Cambridge, USA: Hackett. [Englisch]

LPE 1993 „Notes for Lectures on ,Private Experience' and ,Sense Data““. In: Ludwig Wittgenstein. Philosophical Occasions 1912-1951 S. 200-288. Hg. und eingeleitet von David G. Stern. Indianapolis and Cambridge, USA: Hackett. [Englisch und Deutsch mit englischer Übersetzung]

NPL 1993 „Notes for the ,Philosophical Lecture““. In: Ludwig Wittgenstein. Philosophical Occasions 1912-1951 S. 445-458. Hg. und eingeleitet von David G. Stern. Indianapolis and Cambridge, USA: Hackett. [Englisch]

PH 1993 „Philosophie“. In: Ludwig Wittgenstein. Philosophical Occasions 1912-1951 S. 158-199. Hg. und eingeleitet von James C. Klagge und Alfred Nordmann. Indianapolis and Cambridge, USA: Hackett. [Deutsch mit englischer Übersetzung] 
UW 1993 „Ursache und Wirkung: Intuitives Erfassen / Cause and Effect: Intuitive Awareness". In: Ludwig Wittgenstein. Philosophical Occasions 1912-1951 S. 370-426. Hg. und eingeleitet von James C. Klagge und Alfred Nordmann. Indianapolis and Cambridge, USA: Hackett. [Englisch und Deutsch mit englischer Übersetzung]

WBG 1993 „Geleitwort zum Wörterbuch für Volksschulen / Preface to the Dictionary for Elementary Schools“. In: Ludwig Wittgenstein. Philosophical Occasions 1912-1951 S. 12-27. Hg. und eingeleitet von James C. Klagge und Alfred Nordmann. Indianapolis and Cambridge, USA: Hackett. [Deutsch mit englischer Übersetzung]

1261993 In: Nedo, Michael (1993). Ludwig Wittgenstein: Wiener Ausgabe. Einführung/Introduction S. 61-71. Wien, New York: Springer. [Edition der Seiten 116-133 von MS 126 und dreier „Facsimileseiten aus Wittgensteins Exemplar von G.H. Hardys A Course of Pure Mathematics mit Randbemerkungen Wittgensteins". Deutsch und Englisch]

1151993 In: Nedo, Michael (1993). Ludwig Wittgenstein: Wiener Ausgabe. Einführung/Introduction S. 76-77 und 92-93. Wien, New York: Springer. [Edition der Seiten 118 und 129 von MS 115 mit Facsimiles. Deutsch]

1051993 In: Nedo, Michael (1993). Ludwig Wittgenstein: Wiener Ausgabe. Einführung/Introduction S. 105-121. Wien, New York: Springer. [Edition der Seiten 1, 3, 5, 2, 4 und Rectoseiten 7-35 von MS 105 mit Facsimiles. Deutsch]

VB 1994 Vermischte Bemerkungen. Hg. von G.H. von Wright unter Mitarbeit von Heikki Nyman. Neubearbeitung des Textes durch Alois Pichler. Frankfurt am Main: Suhrkamp. [Enthält ein bisher unveröffentlichtes Gedicht von Wittgenstein. Mit Registern und einem textkritischen Anhang. Deutsch. Erste Veröffentlichung auf Englisch VB 1998. Neubearbeitung von Wittgensteins Graphik durch Michael A.R. Biggs]

1151996 „115 Volume XI. „Philosophische Bemerkungen“. In: Machine-readable version text samples http://www.hit.uib.no/ wab/sample/www.htm. Hg. vom Wittgenstein-Archiv an der Universität Bergen unter der Leitung von Claus Huitfeldt. [Web-Ausgabe von MS 115 in „normalized“ und „diplomatic transcriptions“. Deutsch. Neubearbeitung von Wittgensteins Graphik durch Michael A.R. Biggs] 
2011996 „201a „Notes on Logic“. In: Machine-readable version text samples http://www.hit.uib.no/wab/sample/www.htm. Hg. vom Wittgenstein-Archiv an der Universität Bergen unter der Leitung von Claus Huitfeldt, in Zusammenarbeit mit Michael A.R. Biggs. [Web-Ausgabe von TS 201 in „normalized“ und „diplomatic transcriptions“. Englisch. Neubearbeitung von Wittgensteins Graphik durch Michael A.R. Biggs]

$2011996 b$ In: Biggs, Michael A.R. (1996). Editing Wittgenstein’s, Notes on Logic"Vol. 2.Bergen: Working Papers from the Wittgenstein Archives at the University of Bergen 11. [Edition von TS 201a in „normalized“ und „diplomatic transcriptions“. Englisch. Neubearbeitung von Wittgensteins Graphik durch Michael A.R. Biggs]

1331996 In: Rothhaupt, Josef G.F. (1996). Farbthemen in Wittgensteins Gesamtnachlaß. Philologisch-philosophische Untersuchungen im Längsschnitt und in Querschnitten. S. 580-588. Weinheim: Beltz Athenäum. [Edition der Seiten 31-68 von MS 133. Deutsch]

1361996 In: Rothhaupt, Josef G.F. (1996). Farbthemen in Wittgensteins Gesamtnachlaß. Philologisch-philosophische Untersuchungen im Längsschnitt und in Querschnitten. S. 588-592. Weinheim: Beltz Athenäum. [Edition der Seiten 92a-99a von MS 136. Deutsch]

1371996 In: Rothhaupt, Josef G.F. (1996). Farbthemen in Wittgensteins Gesamtnachlaß. Philologisch-philosophische Untersuchungen im Längsschnitt und in Querschnitten. S. 592-600. Weinheim: Beltz Athenäum. [Edition der Seiten 4b-9b und 99a-101b von MS 137. Deutsch]

1671996 In: Rothhaupt, Josef G.F. (1996). Farbthemen in Wittgensteins Gesamtnachlaß. Philologisch-philosophische Untersuchungen im Längsschnitt und in Querschnitten. S. 596-600. Weinheim: Beltz Athenäum. [Edition der Seiten 30-35 von MS 167. Deutsch]

1731996 In: Rothhaupt, Josef G.F. (1996). Farbthemen in Wittgensteins Gesamtnachlaß. Philologisch-philosophische Untersuchungen im Längsschnitt und in Querschnitten. S. 602-719. Weinheim: Beltz Athenäum. [Faksimile von den ersten 100 Seiten von MS 173]

1761996 In: Rothhaupt, Josef G.F. (1996). Farbthemen in Wittgensteins Gesamtnachlaß. Philologisch-philosophische Untersuchungen im 
Längsschnitt und in Querschnitten. S. 602-719. Weinheim: Beltz Athenäum. [Faksimile der Seiten 1r-22r von MS 176]

2131996 In: Rothhaupt, Josef G.F. (1996). Farbthemen in Wittgensteins Gesamtnachlaß. Philologisch-philosophische Untersuchungen im Längsschnitt und in Querschnitten. S. 564-578. Weinheim: Beltz Athenäum. [Faksimile der Seiten 51-52 und 473-485 von TS 213]

PT 1996 Prototractatus. An early version of Tractatus Logico-Philosophicus. Edited by B.F. McGuinness, T. Nyberg and G.H. von Wright, with a translation by D.F. Pears and B.F. McGuinness, an historical introduction by G.H. von Wright and a facsimile of the author's manuscript. London: Routledge. [Mit Tabellen zu Entsprechungen zwischen „Prototractatus“ und „Tractatus“ und "Note on variations between the different editions of the Tractatus". Englisch und Deutsch mit englischer Übersetzung. „Preface to 1996 edition“ von B.F. McGuinness. Neubearbeitung von Wittgensteins Graphik durch Michael A.R. Biggs]

DB 1997a Denkbewegungen. Tagebücher 1930-1932, 1936-1937(MS 183). Hg. von Ilse Somavilla. Teil 1: Normalisierte Fassung. Innsbruck: Haymon. [Deutsch]

DB 1997b Denkbewegungen. Tagebücher 1930-1932, 1936-1937 (MS 183). Hg. von Ilse Somavilla. Teil 2: Diplomatische Fassung. Innsbruck: Haymon. [Deutsch]

PI 1997 Philosophical Investigations / Philosophische Untersuchungen. Hg. von G.E.M. Anscombe und R. Rhees, übersetzt von G.E.M. Anscombe. Oxford: Blackwell. [Deutsch mit englischer Übersetzung. Mit einem Register von Garth Hallett. Neubearbeitung von Wittgensteins Graphik durch Michael A.R. Biggs]

VB 1998 Ludwig Wittgenstein Vermischte Bemerkungen. Eine Auswahl aus dem Nachlaß/Culture and Value. A Selection from the Posthumous Remains. Hg. von Georg Henrik von Wright unter Mitarbeit von Heikki Nyman. Neubearbeitung des Textes durch Alois Pichler. Übersetzt von Peter Winch. Revised second edition. Blackwell: Oxford. [Mit Registern. Deutsch mit englischer Übersetzung. Neubearbeitung von Wittgensteins Graphik durch Michael A.R. Biggs]

TB 1998 „Notebooks 1914-1916“. In: Notebooks 1914-1916 S. 2-91. Hg. von G.H. von Wright und G.E.M. Anscombe, 
Bibliographie der deutsch- und englischsprachigen Wittgenstein-Ausgaben

übersetzt von G.E.M. Anscombe. Second edition. Oxford: Basil Blackwell. [Mit einem Register von E.D. Klemke und Ali Enayat. Deutsch mit englischer Übersetzung. Neubearbeitung von Wittgensteins Graphik durch Michael A.R. Biggs]

PB 1998 Philosophical Remarks. Hg. von Rush Rhees, übersetzt von Raymond Hargreaves und Roger White. Oxford: Basil Blackwell. [Mit einem ausführlichen Inhaltsverzeichnis und Corrigenda zu PB 1964. Englische Übersetzung. Neubearbeitung von Wittgensteins Graphik durch Michael A.R. Biggs]

Z 1998 Zettel. Hg. von G.E.M. Anscombe und G.H. von Wright, übersetzt von G.E.M. Anscombe. Second edition. Oxford: Basil Blackwell. [Mit einem Register von Stephen Amdur. Englische Übersetzung. Neubearbeitung von Wittgensteins Graphik durch Michael A.R. Biggs]

LW 1998 Last Writings on the Philosophy of Psychology / Letzte Schriften über die Philosophie der Psychologie. Bd. 1. Hg. von G.H. von Wright und Heikki Nyman, übersetzt von C.G. Luckhardt und Maximilian A.E. Aue. Oxford: Basil Blackwell. [Mit einem Register. Deutsch mit englischer Übersetzung. Neubearbeitung von Wittgensteins Graphik durch Michael A.R. Biggs]

RPP 1998a Remarks on the Philosophy of Psychology/Bemerkungen über die Philosophie der Psychologie. Bd. 1. Hg. von G.E.M. Anscombe und G.H. von Wright, übersetzt von G.E.M. Anscombe. Oxford: Basil Blackwell. [Mit einem Register von Heikki Nyman. Deutsch mit englischer Übersetzung. Neubearbeitung von Wittgensteins Graphik durch Michael A.R. Biggs]

RPP 1998b Remarks on the Philosophy of Psychology/Bemerkungen über die Philosophie der Psychologie. Bd. 2. Hg. von G.H. von Wright und Heikki Nyman, übersetzt von C.G. Luckhardt und Maximilian A.E. Aue. Oxford: Basil Blackwell. [Mit einem Register und Tabellen von Entsprechungen zwischen TS 232 und Z, TS 232 und PI, TS 232 und VB. Deutsch mit englischer Übersetzung. Neubearbeitung von Wittgensteins Graphik durch Michael A.R. Biggs]

PU 2001 Philosophische Untersuchungen. Kritisch-genetische Edition. Hg. von Joachim Schulte in Zusammenarbeit mit Heikki Nyman, Eike von Savigny und Georg Henrik von Wright. Frankfurt am Main: Suhrkamp. [Deutsch] 
PU 2003 Philosophische Untersuchungen. Auf der Grundlage der kritisch-genetischen Edition neu hg. von Joachim Schulte. Frankfurt am Main: Suhrkamp. [Deutsch. Mit einem Nachwort des Herausgebers]

TLP 2003 Logisch-philosophische Abhandlung. Frankfurt am Main: Suhrkamp. [Deutsch]

TLP 2004 Ludwig Wittgensteins "Logisch Philosophische Abhandlung“. Entstehungsgeschichte und Herausgabe der Typoskripte und Korrekturexemplare. Hg. von Gerd Graßhoff und Timm Lampert. Wien, New York: Springer. [Deutsch]

PG 2005 Philosophical Grammar. Hg. von Rush Rhees, übersetzt von Anthony Kenny. 2. Aufl. Berkeley (Calif.), Los Angeles, London: University of California Press. [Englisch]

BT 2005 The Big Typeskript: TS 213. Hg. und übersetzt von C. Grant Luckhardt und Maximilian A.E. Aue. Malden (Mass.), Oxford, Carlton (Vic): Blackwell. [German-English scholars' Edition. Deutsch mit englischer Übersetung]

PU 2006 Philosophische Untersuchungen. Auf der Grundlage der kritisch-genetischen Edition neu hg. von Joachim Schulte. 3. Aufl. Frankfurt am Main: Suhrkamp. [Deutsch]

VB 2006 Ludwig Wittgenstein Vermischte Bemerkungen. Eine Auswahl aus dem Nachlaß/Culture and Value. A Selection from the Posthumous Remains. Hg. von Georg Henrik von Wright unter Mitarbeit von Heikki Nyman. Neubearbeitung des Textes durch Alois Pichler. Übersetzt von Peter Winch. 9. Aufl. Oxford: Blackwell. [Deutsch mit englischer Übersetzung] ÜG 2006 Über Gewißheit. Hg. von G.E.M. Anscombe und G.H. von Wright. 11. Aufl. Frankfurt am Main: Suhrkamp. [Deutsch]

ROC 2007 Remarks on Colour / Bemerkungen über die Farben. Hg. von G.E.M. Anscombe, übersetzt von Linda L. McAlister und Margarete Schättle. 2. Aufl. Berkeley (Calif.), Los Angeles: University of California Press. [Deutsch mit englischer Übersetzung]

PI 2007 Philosophical Investigations. Translated by G.E.M. Anscombe. 6. Aufl. Oxford: Blackwell. [Deutsch mit englischer Übersetzung]

LE 2007 Lecture on ethics. Introduction, Interpretation and Complete Text. Edited by Edoardo Zamuner, E. Valentina Di Lascio and David Levy. With Notes by Ilse Somavilla. QuodlibeT: Macerata [Englisch] 
PI 2009 Philosophical Investigations / Philosophische Untersuchungen. Hg. Von P.M.S. Hacker und Joachim Schulte, übersetzt von G.E.M. Anscombe, P.M.S. Hacker und Joachim Schulte. New York: Wiley. [Deutsch mit englischer Übersetzung]

TLP 2009 Tractatus logico-philosophicus / Logisch-philosophische Abhandlung. Neuauflage. Frankfurt am Main: Suhrkamp. [Deutsch] PIP 2010 Wittgenstein's 1938 Preface. Edited by Nuno Venturinha. In: Wittgenstein after his Nachlass S. 182-188. Edited by Nuno Venturinha. Houndmills: Palgrave Macmillan. [Englisch]

2.3 Veröffentlichungen mit Quellen in den Nachlassteilen 301-311 (Diktate)

BBB 1958 Preliminary Studies for the „Philosophical Investigations“. Generally Known as The Blue and Brown Books. Oxford: Basil Blackwell. [Englisch. Das „Blue Book“ und Teile des „Brown Book" zuerst veröffentlicht auf Deutsch in BBB 1970]

BBB 1960 Preliminary Studies for the „Philosophical Investigations". Generally Known as The Blue and Brown Books. New York: Harper Torchbooks. [Englisch. Dank an Mauro Engelmann, Brian Rogers und David G. Stern für Informationen zu dieser Ausgabe]

AM 1960 „Aufzeichnungen, die G.E. Moore in Norwegen nach Diktat niedergeschrieben hat". Hg. von G.E.M. Anscombe und G.H. von Wright, übersetzt von Günther Patzig und Eberhard Bubser. In: Schriften Bd. 1 S. 226-253. Frankfurt am Main: Suhrkamp. [Englisch mit deutscher Übersetzung]

AM 1961 „Notes dictated to G.E. Moore in Norway“. In: Notebooks 1914-1916 S. 107-118. Hg. von G.H. von Wright und G.E.M. Anscombe, übersetzt von G.E.M. Anscombe. Oxford: Basil Blackwell. [Englisch]

BBB 1969 Preliminary Studies for the "Philosophical Investigations“. Generally Known as The Blue and Brown Books. Second edition. Oxford: Basil Blackwell. [Mit einem Register. Englisch]

BBB 1970 „Das Blaue Buch“ und „Eine Philosophische Betrachtung“. In: Schriften Bd. 5 S. 7-282. Hg. von Rush Rhees, übersetzt von Petra von Morstein. Frankfurt am Main: Suhrkamp. [Deutsche Übersetzung und Deutsch] 
AM 1979 „Notes dictated to G.E. Moore in Norway“. In: Notebooks 1914-1916 S. 108-119. Hg. von G.H. von Wright und G.E.M. Anscombe, übersetzt von G.E.M. Anscombe. Second edition. Oxford: Basil Blackwell. [Mit einem Register von E.D. Klemke und Ali Enayat. Englisch]

YB 1979 „The Yellow Book (Selected Parts)“. In: Wittgenstein's Lectures: Cambridge, 1932-1935 S. 41-73. Hg. von Alice Ambrose.

Oxford: Basil Blackwell. [Englisch. Erste Veröffentlichung auf Deutsch in YB 1984]

YB 1982 „The Yellow Book (Selected Parts)“. In: Wittgenstein's Lectures: Cambridge, 1932-1935 S. 41-73. Hg. von Alice Ambrose.

Oxford: Basil Blackwell. [Mit einem Register. Englisch. Erste Veröffentlichung auf Deutsch YB 1984]

YB 1984 „Das Gelbe Buch. 1933/34 (Eine Auswahl)“. Hg. von Alice Ambrose, übersetzt von Joachim Schulte. In: Vorlesungen 1930-1935 S. 199-241. Frankfurt am Main: Suhrkamp. [Mit einem Register. Deutsche Übersetzung]

BBB 2000 Preliminary Studies for the "Philosophical Investigations“. Generally Known as The Blue and Brown Books. Second edition. Oxford: Basil Blackwell. [Mit einem Register. Englisch. Neubearbeitung von Wittgensteins Graphik durch Michael A.R. Biggs]

2.4 Veröffentlichungen von Vorlesungs- und Gesprächsaufzeichnungen (Auswahl)

MWL 1954a Moore, G.E. „Wittgenstein's Lectures in 1930-33“(Part I). In: Mind 63 S. 1-15 (Edinburgh 1954). [Englisch]

MWL 1954b Moore, G.E. „Wittgenstein's Lectures in 1930-33“(Part II). In: Mind 63 S. 289-316 (Edinburgh 1954). [Englisch]

MWL 1955a Moore, G.E. „Wittgenstein's Lectures in 1930-33“ (Part III). In: Mind 64 S. 1-27 (Edinburgh 1955). [Englisch]

MWL 1955b Moore, G.E. „Two Corrections“. In: Mind 64 p.264 (Edinburgh 1955). [Englisch]

MAM 1958 Malcolm, N. Ludwig Wittgenstein: A Memoir. With a Biographical Sketch by G.H. von Wright. London: Oxford University Press. [Englisch. Erste Veröffentlichung auf Deutsch MAM 1961] 
MAM 1961 Malcolm, M. Ludwig Wittgenstein - Ein Erinnerungsbuch. Mit einer biographischen Skizze von G.H. von Wright.

Übersetzt von C. Frank und A. Sjögren. München: R. Oldenbourg. [Deutsche Übersetzung]

FWS 1964 „Aus F. Waismanns stenographischer Abschrift von Reden und Gesprächen Wittgensteins zwischen Dezember 1929 und September 1931“. In: Philosophische Bemerkungen S. 315-346. Hg. von Rush Rhees. Oxford: Basil Blackwell. [Deutsch. Veröffentlicht in Schriften Bd. 2 im selben Jahr. Erste Veröffentlichung auf Englisch FWS 1975]

FWN 1965 Waismann, F. „Notes on Talks with Wittgenstein“. In: "Wittgenstein's Lecture on Ethics“. In: The Philosophical Review 74 S. 12-16 (Ithaca, USA, 1965). Übersetzt von Max Black. [Deutsch mit englischer Übersetzung]

RRD 1965 Rhees, R. „Some Developments in Wittgenstein's View of Ethics“. In: „Wittgenstein's Lecture on Ethics“. In: The Philosophical Review 74 S. 17-26 (Ithaca, USA, 1965). [Englisch]

AWU 1966 Ambrose, A. „Wittgenstein on Universals“. In: Essays in Analysis S. 104-121. London: George Allen and Unwin. [Englisch]

LA 1966 Lectures and Conversations. Hg. von Cyril Barrett. Oxford: Basil Blackwell. [Englisch. Erste Veröffentlichung auf Deutsch LA 1968]

WWK 1967 Ludwig Wittgenstein und der Wiener Kreis. Hg. von Brian McGuinness. Oxford: Basil Blackwell. [Mit einem Register. Deutsch. Veröffentlicht als Schriften Bd. 3 im selben Jahr. Erste Veröffentlichung auf Englisch WWK 1979]

LA 1968 Vorlesungen und Gespräche über Ästhetik, Psychologie und Religion. Hg. von Cyril Barrett, übersetzt und eingeleitet von Eberhard Bubser. Göttingen: Vandenhoeck und Ruprecht. [Deutsche Übersetzung]

RRC 1970 „On Continuity: Wittgenstein’s Ideas 1938“. In: Rhees, R. Discussions of Wittgenstein S. 104-157. London: Routledge and Kegan Paul. [Englisch]

FWS 1975 „From F. Waismann's shorthand transcript of Wittgenstein's talks and conversation between December 1929 and September 1931“. In: Philosophical Remarks S. 315-346. Hg. von Rush Rhees, übersetzt von Raymond Hargreaves und 
Roger White. Oxford: Basil Blackwell. [Englische Übersetzung]

LFM 1976 Wittgenstein's Lectures on the Foundations of Mathematics: Cambridge, 1939. Hg. von Cora Diamond. Ithaca, USA: Cornell University Press. [Mit einem Register. Englisch. Erste Veröffentlichung auf Deutsch LFM 1978]

MDN 1976 Drury, M.O'C. „Some Notes on Conversations with Wittgenstein“. In: „Essays on Wittgenstein in Honour of G.H. von Wright“. In: Acta Philosophica Fennica 28 S. 22-40 (Amsterdam, Holland, 1976). [Englisch. Erste Veröffentlichung auf Deutsch MDN 1987]

UW 1976 „Ursache und Wirkung: Intuitives Erfassen / Cause and Effect: Intuitive Awareness". Hg. von Rush Rhees, übersetzt von Peter Winch. In: Philosophia 6 S. 392-445 (RamatGan, Israel 1976). [Englisch und Deutsch mit englischer Übersetzung. Erste Veröffentlichung auf Deutsch UW 1989]

OH 1977 Murray, M. „On Heidegger on Being and Dread“. In: Murray, M. Heidegger and Modern Philosophy S. 80-83. New Haven, USA: Yale University Press. [Englische Übersetzung. Erste Veröffentlichung auf Deutsch in WWK 1967 S. 68-69]

LFM 1978 „Wittgensteins Vorlesungen über die Grundlagen der Mathematik. Cambridge, 1939“. In: Schriften Bd. 7. Hg. von Cora Diamond, übersetzt von Joachim Schulte. Frankfurt am Main: Suhrkamp. [Mit einem Register. Deutsche Übersetzung]

AWL 1979 Wittgenstein's Lectures: Cambridge, 1932-1935. Hg. von Alice Ambrose. Oxford: Basil Blackwell. [Englisch. Erste Veröffentlichung auf Deutsch in AWL 1984]

WWK 1979 Ludwig Wittgenstein and the Vienna Circle. Conversations recorded by Friedrich Waismann. Hg. von Brian McGuinness, übersetzt von Joachim Schulte und Brian McGuinness. Oxford: Basil Blackwell. [Mit einem Register. Englisch und englische Übersetzung]

LWL 1980 Wittgenstein's Lectures: Cambridge, 1930-1932. Hg. von Desmond Lee. Oxford: Basil Blackwell. [Mit einem Register. Englisch. Erste Veröffentlichung auf Deutsch LWL 1984]

MDC 1981 M.O'C. Drury. „Conversations with Wittgenstein“. In: Recollections of Wittgenstein S. 112-189. Hg. von R. Rhees. 
Oxford: Basil Blackwell. [Mit einem Register. Englisch. Erste Veröffentlichung auf Deutsch MDC 1987]

AWL 1982 Wittgenstein's Lectures: Cambridge, 1932-1935. Hg. von Alice Ambrose. Oxford: Basil Blackwell. [Mit einem Register. Englisch. Erste Veröffentlichung auf Deutsch AWL 1984]

AWL 1984 „Cambridge 1932-1935“. Hg. von Alice Ambrose, übersetzt von Joachim Schulte. In: Ludwig Wittgenstein. Vorlesungen 1930-1935 S. 141-442. Frankfurt am Main:

Suhrkamp. [Mit einem Register. Deutsche Übersetzung]

LWL 1984 „Cambridge 1930-1932“. Hg. von Desmond Lee, übersetzt von Joachim Schulte. In: Ludwig Wittgenstein. Vorlesungen 1930-1935 S. 9-139. Frankfurt am Main: Suhrkamp. [Mit einem Register. Deutsche Übersetzung]

MAM 1984 Malcolm, N. Ludwig Wittgenstein: A Memoir. With a Biographical Sketch by G.H. von Wright and Wittgenstein's letters to Malcolm. Second edition. London: Oxford University Press. [Mit einem Register. Englisch. Erste Veröffentlichung auf Deutsch MAM 1987]

RSD 1984a Rhees, R.: „The Language of Sense Data and Private Experience-I“. In: Philosophical Investigations 7 S. 1-45 (Oxford, 1984). [Englisch]

RSD 1984b Rhees, R.: „The Language of Sense Data and Private Experience-II“. In: Philosophical Investigations 7 S. 101-140 (Oxford, 1984). [Englisch]

OBC 1986 Bouwsma, O.K.: Wittgenstein: Conversations 1949-1951. Hg. und eingeleitet von J.L. Craft und R.E. Hustwit. Indianapolis, USA: Hackett. [Englisch]

MAM 1987 Malcolm, M. Erinnerungen an Wittgenstein. Mit einer biographischen Skizze von Georg Henrik von Wright und Wittgensteins Briefen an Norman Malcolm. Übersetzt von Claudia Frank und Joachim Schulte. Frankfurt: Suhrkamp am Main. [Mit einem Register. Deutsche Übersetzung]

MDC 1987 Drury, M.O’C. „Gespräche mit Wittgenstein“. In: Ludwig Wittgenstein: Porträts und Gespräche S. 142-235. Hg. von Rush Rhees, mit einer Einleitung von Norman Malcolm, übersetzt von Joachim Schulte. Frankfurt am Main: Suhrkamp. [Deutsche Übersetzung]

MDN 1987 Drury, M.O’C. „Bemerkungen zu einigen Gesprächen mit Wittgenstein“. In: Ludwig Wittgenstein: Porträts und Ge- 
PGL 1988 Wittgenstein's Lectures on Philosophical Psychology 19461947. Hg. von P.T. Geach. New York, USA: Harvester. [Englisch. Erste Veröffentlichung auf Deutsch PGL 1991]

UW 1989 „Ursache und Wirkung: Intuitives Erfassen“. In: Vortrag über Ethik S. 101-139. Hg. und übersetzt von Joachim Schulte. Frankfurt am Main: Suhrkamp. [Deutsch und deutsche Übersetzung]

YSF 1989 „A Lecture on Freedom of the Will“. In: Philosophical Investigations 12 S. 85-100 (Oxford 1989). [Englisch]

PGL 1991 Ludwig Wittgenstein. Vorlesungen über die Philosophie der Psychologie 1946-1947. Hg. von P.T. Geach, übersetzt von Joachim Schulte. Frankfurt am Main: Suhrkamp. [Mit einem Register. Deutsche Übersetzung]

MWL 1993 Moore, G.E. „Wittgenstein’s Lectures in 1930-33“. In: Ludwig Wittgenstein. Philosophical Occasions 1912-1951 S. 45-114. Hg. und eingeleitet von James C. Klagge und Alfred Nordmann. Indianapolis and Cambridge, USA: Hackett. [Englisch]

RSD 1993 Rhees, R. „The Language of Sense Data and Private Experience". In: Ludwig Wittgenstein. Philosophical Occasions 1912-1951 S. 289-367. Hg. und eingeleitet von James C. Klagge und Alfred Nordmann. Indianapolis and Cambridge, USA: Hackett. [Englisch]

UW 1993 „Ursache und Wirkung: Intuitives Erfassen / Cause and Effect: Intuitive Awareness". In: Ludwig Wittgenstein. Philosophical Occasions 1912-1951 S. 370-426. Hg. und eingeleitet von James C. Klagge und Alfred Nordmann. Indianapolis and Cambridge, USA: Hackett. [Englisch und Deutsch mit englischer Übersetzung]

YSF 1993 „Lectures on Freedom of the Will (Notes by Yorick Smythies)“. In: Ludwig Wittgenstein. Philosophical Occasions 1912-1951 S. 427-444. Hg. und eingeleitet von James C. Klagge und Alfred Nordmann. Indianapolis and Cambridge, USA: Hackett. [Englisch]

VW 2003 The Voices of Wittgenstein. The Vienna Circle. Hg. von Gordon Baker, übersetzt von Gordon Baker, Michael 
Mackert, John Connolly und Vasilis Politis. London, New York: Routledge: 2003. [Enthält Diktate von Wittgenstein an Waismann und überarbeitete Texte von Waismann. Deutsch mit englischer Übersetzung]

VÄ 2005 Vorlesungen und Gespräche über Ästhetik, Psychoanalyse und religiösen Glauben. Zusammengestellt und hg. von Cyril Barrett aus Notizen von Yorick Smythies, Rush Rhees und James Taylor, übersetzt von Ralf Funke. 1. Auflage. Düsseldorf/Bonn: Parerga 1994; 3. Auflage. Frankfurt am Main: Fischer 2005. [Deutsche Übersetzung]

RN 2010 The Ramsey Notes on Time and Mathematics. Edited by Nuno Venturinha, with an English Translation by James M.

Thompson. In: Wittgenstein after his Nachlass S. 173-181. Edited by Nuno Venturinha. Houndmills: Palgrave Macmillan. [Englisch]

2.5 Veröffentlichungen von Korrespondenz (Auswahl)

CAM 1958 Briefe in:Malcolm, N. Ludwig Wittgenstein:AMemoir. With a Biographical Sketch by G.H. von Wright. London: Oxford University Press. [Englisch. Erste Veröffentlichung auf Deutsch CAM 1961]

CBR 1960 „Auszüge aus Wittgensteins Briefen an Russell, 1912-20“. Hg. von G.E.M. Anscombe und G.H. von Wright. Übersetzt von G. Patzig und E. Bubser. In: Schriften Bd. 1 S. 254-278. Frankfurt am Main: Suhrkamp. [Deutsch und Englisch mit deutscher Übersetzung]

CAM 1961 Briefe in: Malcolm, M. Ludwig Wittgenstein - Ein Erinnerungsbuch. Mit einer biographischen Skizze von G.H. von Wright. Übersetzt von C. Frank und A. Sjögren. München: R. Oldenbourg. [Deutsche Übersetzung]

CBR 1961 „Extracts from Wittgenstein's letters to Russell, 1912-20“. In: Notebooks 1914-1916 S. 119-131. Hg. von G.H. von Wright und G.E.M. Anscombe, übersetzt von G.E.M. Anscombe. Oxford: Basil Blackwell. [Englisch und Deutsch mit englischer Übersetzung]

CWE 1963 Eccles, W. „Some Letters of Wittgenstein, 1912-1939“. In: Hermanthea 97 S. 57-65 (Dublin, Eire, 1967). [Englisch] 
CPE 1967 Engelmann, Paul „Briefe von Wittgenstein / Letters from Wittgenstein". In: Letters from Ludwig Wittgenstein. With a MemoirS. 2-59. Hg. von B.F. McGuinness, übersetzt von L. Furtmüller. Oxford: Basil Blackwell. [Mit einem Register. Deutsch mit englischer Übersetzung]

CBR 1968 Briefe in: Russell, B. Autobiography Bd. 2 S. 116-121. London: George Allen and Unwin. [Deutsch mit englischer Übersetzung]

CLF 1969 Briefe an Ludwig von Ficker. Hg. von Georg Henrik von Wright in Zusammenarbeit mit Walter Methlagl. Brenner Studien 1. Salzburg: Otto Müller. [Deutsch. Erste Veröffentlichung auf Englisch CLF 1979]

C 1971 Briefe in: von Wright, G.H. „The Origin of Wittgenstein's Tractatus". In: Prototractatus S. 1-34. London: Routledge and Kegan Paul. [Englisch und Englische Übersetzung]

CCO 1973 Letters to C.K. Ogden. Edited with an Introduction by G.H. von Wright and an Appendix of Letters by Frank Plumpton Ramsey. Oxford: Basil Blackwell und London: Routledge and Kegan Paul. [Mit einem Register. Englisch]

CRK 1974 Letters to Russell, Keynes and Moore. Edited with an Introduction by G.H. von Wright, assisted by B.F. McGuinness. Oxford: Basil Blackwell. [Englisch und Deutsch mit englischer Übersetzung]

CMW 1976 Briefe in: Leitner, Bernhard The Architecture of Ludwig Wittgenstein S. 122-124. New York, USA: New York University Press. [Englisch und Deutsch mit einem Facsimile der Briefe S. 122-123 und einem Facsimile von Wittgensteins Plänen S. 44-46]

CRK 1977 Letters to Russell, Keynes and Moore. Edited with an Introduction by G.H. von Wright, assisted by B.F. McGuinness. Second edition with minor corrections. Oxford: Basil Blackwell. [Mit einem Register von Leo Groarke. Englisch und Deutsch mit englischer Übersetzung]

C 1979a Briefe in: von Wright, Georg Henrik „The Origin of Wittgenstein's Tractatus“. In: Wittgenstein: Sources and Perspectives S. 99-137. Hg. von C.G. Luckhardt. Sussex: The Harvester Press. [Mit Registern. Englisch und englische Übersetzung] 
C 1979b Briefe in: Wuchterl, Kurt und Hübner, Adolf Ludwig Wittgenstein. Hamburg: Rowohlt. [Mit Register. Deutsch und deutsche Übersetzung]

CBR 1979 „Extracts from Wittgenstein's letters to Russell, 1912-20“. In: Notebooks 1914-1916 S. 120-132. Hg. von G.H. von Wright und G.E.M. Anscombe, übersetzt von G.E.M. Anscombe. Oxford: Basil Blackwell. [Mit einem Register von E.D. Klemke und Ali Enayat. Englisch und Deutsch mit englischer Übersetzung]

CLF 1979 „Letters to Ludwig von Ficker“. Hg. von Allan Janik, übersetzt von Bruce Gillette. In: Wittgenstein: Sources and Perspectives S. 82-98. Hg. von C.G. Luckhardt. Sussex: The Harvester Press. [Mit Registern. Englische Übersetzung]

CB 1980 Briefe. Hg. von B.F. McGuinness und G.H. von Wright, übersetzt von J. Schulte. Frankfurt am Main: Suhrkamp. [Mit einem Register zum deutschen Text von Joachim Schulte. Deutsch und Englisch mit deutscher Übersetzung]

C 1983 Briefe in: Wittgenstein. Sein Leben in Bildern und Texten. Hg. von Michael Nedo und Michele Ranchetti. Frankfurt am Main: Suhrkamp. [Deutsch und deutsche Übersetzung]

CVW 1983a „Some Hitherto Unpublished Letters from Ludwig Wittgenstein to Georg Henrik von Wright". In: The Cambridge Review 104 S. 56-64 (Cambridge 1983). [Englisch. Erste Veröffentlichung auf Deutsch CVW 1983a]

CVW 1983b „Briefe an von Wright“. In: Weder-Noch. Tangenten zu den finnisch-österreichischen Kulturbeziehungen. Übersetzt von S. Stössel. Helsinki: Mitteilungen aus der deutschen Bibliothek. [Deutsch. Zitiert nach Schulte 1989:S.237]

CAM 1984 „Wittgenstein's Letters to Norman Malcolm“. In: Malcolm, N. Ludwig Wittgenstein: A Memoir. With a Biographical Sketch by G.H. von Wright and Wittgenstein's Letters to Malcolm S. 85-134. London: Oxford University Press. [Mit einem Register. Englisch]

CLH 1985 Briefe in: Wünsche, K. Der Volksschullehrer Ludwig Wittgenstein S. 295-326. Frankfurt am Main: Suhrkamp. [Deutsch]

CAM 1987 „Wittgensteins Briefe an Norman Malcolm“. In: Malcolm, N. Erinnerungen an Wittgenstein. Mit einer biographischen Skizze von Georg Henrik von Wright und Wittgensteins Briefen an Norman Malcolm S. 137-216. Übersetzt von Joachim 
Schulte. Frankfurt am Main: Suhrkamp. [Mit einem Register. Deutsche Übersetzung]

C 1988a Briefe in: McGuinness, Brian Wittgenstein: A Life. London: Duckworth. [Mit einem Register. Englisch und englische Übersetzung]

C 1988b Briefe in: McGuinness, Brian Wittgensteins frühe Jahre. Übersetzt von Joachim Schulte. Frankfurt am Main: Suhrkamp. [Mit einem Register. Deutsch und deutsche Übersetzung]

CBR 1990 „Unpublished Correspondence between Russell \& Wittgenstein“. In: Russell 10 S. 101-124 (Hamilton, Canada, 1990). Hg. von B.F. McGuinness und G.H. von Wright. [Englisch und Deutsch mit englischer Übersetzung]

C 1990 Briefe in: Monk, Ray Ludwig Wittgenstein. London: Jonathan Cape. [Mit einem Register. Englisch und englische Übersetzung]

CDP 1990 Briefe in: A Portrait of Wittgenstein as a Young Man S. 93-112 Hg. von G.H. von Wright. Oxford: Basil Blackwell. [Mit einem Register. Englisch]

C 1992 Briefe in: Monk, Ray Wittgenstein. Übersetzt von Hans Günter Holl und Eberhard Rathgeb. Stuttgart: Klett-Cotta. [Deutsch und deutsche Übersetzung]

CVW 1993 „Letters from Ludwig Wittgenstein to Georg Henrik von Wright". In: Ludwig Wittgenstein. Philosophical Occasions 1912-1951 S. 459-479. Hg. von James C. Klagge und Alfred Nordmann. Indianapolis and Cambridge, USA: Hackett. [Englisch]

CWE 1993 Eccles, W. „Some Letters of Ludwig Wittgenstein“. In: Ludwig Wittgenstein. Philosophical Occasions 1912-1951 S. 2-11. Hg. von James C. Klagge und Alfred Nordmann. Indianapolis and Cambridge, USA: Hackett. Hermanthea 97 S. 57-65 (Dublin, Eire, 1967). [Englisch]

CSK 1994 „Ludwig Wittgenstein’s Correspondence with Skjolden“. Hg. von Georg Henrik von Wright und Knut Olav Åmås. In:Wittgenstein and Norway. S. 83-162. Hg. von Kjell S. Johannessen, Rolf Larsen, Knut Olav Åmås. Oslo: Solum. [Norwegisch, Deutsch und Englisch und englische Übersetzung]

CLH 1994 Ludwig Hänsel-Ludwig Wittgenstein. Eine Freundschaft. Briefe. Aufsätze. Kommentare. Hg. von Ilse Somavilla, Anton 
Unterkircher und Christian Paul Berger unter Leitung von Walter Methlagl und Allan Janik. Innsbruck: Haymon. [Deutsch]

CC 1995 Cambridge Letters. Correspondence with Russell, Keynes, Moore, Ramsey and Sraffa. Hg. von Brian McGuinness und G.H. von Wright. Oxford, U.K./Cambridge, USA: Blackwell. [Deutsch und Englisch und englische Übersetzung]

CVG 1995 Briefe in: Rothhaupt, Josef G.F. „Ludwig Wittgenstein und Victor Gollancz“. In: Wittgenstein Studies 1/95 Datei 20-195.TXT. Hg. von K.-O. Apel, F. Börncke, N. Garver, B. McGuinness, P. Hacker, R. Haller, W. Lütterfelds, G. Meggle, C. Nyíri, K. Puhl, Th. Rentsch, A. Roser, J.G.F. Rothhaupt, J. Schulte, U. Steinvorth, P. Stekeler-Weithofer, W. Vossenkuhl (Passau). [Englisch]

CF 1996 Familienbriefe. Hg. von Brian McGuinness, Maria Concetta Ascher, Otto Pfersmann. Schriftenreihe der WittgensteinGesellschaft Band 23. Wien: Hölder-Pichler-Tempsky. [Deutsch und Englisch]

PPO 2003 Public and Private Occasions. Hg. von James C. Klagge und Alfred Nordmann. Lanham, Boulder, New York, Oxford: Rowman and Littlefield 2003. [Enthält Wittgensteins Tagebücher der 1930er Jahre (erstmals in englischer Sprache), Wittgensteins Korrespondenz mit Ludwig Hänsel, Vorlesungen in Cambridge und Dokumente im Zusammenhang mit dem Moral Science Club]

LUS 2004 Licht und Schatten. Ein nächtliches (Traum-)Erlebnis und ein Brieffragment. Hg. von Ilse Somavilla. Innsbruck, Wien: Haymon 2004. [Deutsch. Enthält eine tagebuchartige Aufzeichnung eines (Traum-)Erlebnisses aus dem Jahre 1922 und das Fragment eines Briefes, den Wittgenstein vermutlich im Jahre 1925 an seine Schwester Hermine schrieb]

WE 2006 Wittgenstein-Engelmann: Briefe, Begegnungen, Erinnerungen. Hg. von Ilse Somavilla unter Mitarbeit von Brian McGuinness. Innsbruck und Wien: Haymon 2006. [Deutsch]

WC 2008 Wittgenstein in Cambridge. Letters and Documents, 1911 1951. Hg. Von Brian McGuinness. Malden (Mass.):

Blackwell 2008. [Enthält die Korrespondenzen mit Russell, Keynes, Moore und Ramsey sowie 200 bisher nicht-veröffentlichte Briefe und Dokumente, darunter die Korrespon- 
denzen mit Sraffa und mit Wittgensteins Schülern. Deutsch und Englisch]

CS [2010] Moritz Schlick - Wissenschaftlicher Briefwechsel. Hg. von Reinhard Fabian, Mathias Iven und Heiner Rutte. Wien: Springer. [Deutsch. Veröffentlichung in Vorbereitung]

3 Bibliographie der Veröffentlichungen von Waismanns „Wittgenstein-Arbeiten“ (Auswahl)

WLA 1930 „Logische Analyse des Wahrscheinlichkeitsbegriffs“. In: Erkenntnis 1 S.228-248 (Dordrecht 1930/31).

WBI 1936 „Über den Begriff der Identität“. In: Erkenntnis 6 S.56-64 (Dordrecht 1936).

WMD 1936 Einfuihrung in das mathematische Denken. Wien: Gerold \& Co.

WMD 1951 Introduction to Mathematical Thinking. Übersetzt von T.J. Benac. London: Hafner.

WLP 1965 The Principles of Linguistic Philosophy. Hg. von R. Harré. London/New York: Macmillan/St. Martin's Press.

WLP 1976 Logik, Sprache, Philosophie. Mit einer Vorrede von Moritz Schlick hg. von Gordon P. Baker und Brian McGuinness unter Mitwirkung von Joachim Schulte. Stuttgart: Philipp Reclam Jun. 